Pak. j. sci. ind. res. Ser. B: biol. sci. 2017 60(3) 132-140

\title{
Mutagenic Effects on the Growth, Reproductive and Yield Parameters of Praecitrullus fistulosus
}

\author{
Mehreen Khan, Muhammad Rafiq*, Syed Habib Ahmed Naqvi, \\ Naseem Khatoon and Muhammad Umar Dahot \\ Institute of Biotechnology and Genetic Engineering, University of Sindh, Jamshoro, Pakistan
}

(received June 2, 2016; revised December 11, 2016; accepted December 13, 2016)

\begin{abstract}
The aim of this study was to modify the growth, reproductive and yield parameters of Praecitrullus fistulosus by mutagenesis. The seeds of the plant were treated with chemical mutagens including ethidium bromide and colchicine in concentration of $(0.05$ and $0.10 \%)$ and $(0.01$ and $0.02 \%)$, respectively. Seeds were also treated with UV rays at periods of 1 and $2 \mathrm{~h}$ and X-rays for $75 \mathrm{KeV}$ at periods of 5 and 10 sec. The growth features were observed at an interval of one week till the $11^{\text {th }}$ week of their growth period. The data showed that seed germination value and lethality (\%) of plants were 80 and $75 \%$, respectively and highest in control plants. The time of seed germination (1.6 days) was least in plants treated with $\mathrm{X}$-rays $(10 \mathrm{sec})$. The mutation frequency $(80 \%)$ was found to be highest in X-rays $(10 \mathrm{sec})$ and colchicine $0.02 \%$. The vegetative growth parameters such as stem length $(77 \mathrm{~cm})$, length of leaves $(6.6 \mathrm{~cm})$, average number of leaves (36) and leaf surface area $\left(49.6 \mathrm{~cm}^{2}\right)$ was highest against ethidium bromide $0.01 \%$, colchicine $0.02 \%$, ethidium bromide $0.10 \%$ and UV rays $1 \mathrm{~h}$, respectively. While the average diameter of stem of control plants was highest $\left(49.6 \mathrm{~cm}^{2}\right)$. The minimum flowering time (31 days) and fruiting time (42.5 days) were observed in ethidium bromide $0.05 \%$ and colchicine $0.01 \%$ treated plants. The highest number of fruits (4) was observed in colchicine $0.01 \%$ treated plants. In conclusion, seeds of $P$. fistulosus treated with ethidium bromide and colchicine caused positive impact on growth, reproduction and yield attributes as compared to UV and X-rays treatments.
\end{abstract}

Keywords: mutagenic effects, physical mutagens, chemical mutagens, Praecitrullus fistulosus

\section{Introduction}

Plants are rich source of secondary metabolites that are known to play major role in survival and adaptation of plants to their environment (Bourgaud et al., 2001). There are about 200,000 plant secondary metabolites that provide defense mechanism to the plants (Adamu and Aliyu, 2007). World population is increasing at an alarming rate therefore, the food demands have overcome the available land resources. Along with other food sources, vegetables are also considered as alternate inexpensive source of energy for the population of developing countries (Akwaowo et al., 2000). Vegetables are rich in biochemicals and nutrients like carbohydrates, protein, vitamins, ascorbic acid, calcium, iron and substantial amount of trace elements (Jimoh and Oladiji, 2005; Prakash and Pal, 1991).

Praecitrullus fistulosus is an annual Cucurbitaceae plant (Tyagi et al., 2012) that has been cultivated in Asia since earlier periods and grown mainly in India and Pakistan (Levi et al., 2010). P. fistulosus is locally called 'tinda' that has tender fruits which are picked during

*Author for correspondence; E-mail: m.rafiq@usindh.edu.pk immature stage and used as cooked vegetable (Sujatha and Seshadri, 1989). P. fistulosus plant is also rich in many nutritive properties (Gautam et al., 2011), antiinflammatory, anti-tumorous, antidiabetic, antioxidant, anthelminthic, and other therapeutic potential for microbes as an antibacterial agent (Dixit and Kar, 2010).

Food and crop production should be increased at least at the same rate or even much faster rate than world population. In order to improve crop production and enhancement of their economic and agronomic characteristics, mutagenesis can be used (Tah and Roychowdhury, 2011). Mutagenesis is widely used method for crop improvement and variety production (Chopra, 2005). More than 70 decades, mutagenic agents have been employed to induce many different phenotypic traits and variation in plants (Kharkwal and Shu, 2009). Treatment with mutagens may break or rearrange chromosomes by modifying order of genes depending upon the nature of mutagen used in experimental study and do not show any phenotypic expression (Adamu and Aliyu, 2007). Different chemical and physical mutagens are used to induce mutation. Colchicine (COL) is a compound that can cause dynamic instability in 
microtubule formation during mitosis (Ravelli et al., 2004). Colchicine has been used widely in cultures to change the ploidy level (Van Harten, 1998). Ethidium bromide $(\mathrm{EtBr})$ is an intercalating molecule that binds by intercalation between the base pairs of DNA strands and is extremely useful tool in different techniques (Palchaudhuri and Hergenrother, 2007). The use of radiation on plants is a wide and complex field and has been applied on a large number of plant species. Radiation affects the germination, growth, development, weight and yield parameters of plants (Sameh et al., 2006; Song et al., 2006). Ultraviolet radiations (UV) are efficiently absorbed by DNA molecules that causes formation of covalent linkages between pyrimidine bases, hence resulting in the formation of photo dimers (Taylor, 2006), causing blockage of replication as well as transcription (Chopra, 2005). UV-C radiations are shortest wavelength (100-280 nm) with the higher associated energy (Katerova et al., 2009) and effects extremely germicidal actions on microorganisms in air, on surfaces and in water (Siddiqui et al., 2011).

X-rays penetrate deeper in to the tissues (Amano, 2006) and involves in the production of clusters in DNA that causes damages (Sutherland et al., 2000). The effect of $\mathrm{X}$-rays dose on animals and plants has been studied by Yang et al. (2011). Different growth regulators can be used such as GABA in order to enhance morphological and yield characters (Islam et al., 2010) in addition to increased levels of nitrogen gas and zinc sulphate (Asif et al., 2013). In Pakistan, a very little work has been done for the improvement of vegetables germplasm. As $P$. fistulosus contains excellent antioxidant activity as compared to other cucurbits along with antidiabetic and anti-cancerous activities. The primary objective of this study was therefore, to determine effect of colchicine, ethidium bromide, UV and X rays on growth, development and yield attributes.

\section{Materials and Methods}

The experiments were carried out during April-October 2014 at the experimental field of Institute of Biotechnology and Genetic Engineering, University of Sindh, Jamshoro, Sindh, Pakistan. Praecitrullus fistulosus (tinda seeds) were obtained from private seed company, Hyderabad, Sindh, Pakistan.

Treatment of $P$. fistulosus seeds with chemical mutagens. For induced mutation with chemical mutagens, $0.05 \%$ $(0.05 \mathrm{~g} / 100 \mathrm{~mL})$ and $0.10 \%(0.1 \mathrm{~g} / 100 \mathrm{~mL})$ of ethidium bromide (ACROS) and $0.01 \%(0.01 \mathrm{~g} / 100 \mathrm{~mL})$ and $0.02 \%(0.02 \mathrm{~g} / 100 \mathrm{~mL})$ of colchicine (ACROS) were prepared. Briefly, for each treatment fifty healthy, dry and uniform sized seeds were initially pre-soaked in $100 \mathrm{~mL}$ distilled water at room temperature for two hours. After removing the excess water, seeds were submerged in $100 \mathrm{~mL}$ solutions containing ethidium bromide and colchicine separately for one hour in the dark with gentle shaking at room temperature (Koornneef et al., 1982). The treated seeds were washed in running water to remove excess chemical mutagens and were sowed in the soil. The experiment was repeated for two times thus 150 seeds were used for each treatment.

Treatment of $\boldsymbol{P}$. fistulosus seeds with physical mutagens. Seeds were initially placed in a desiccator with $70 \%$ glycerol for two days for moisture equilibration to about $15-20 \%$. For each treatment, fifty healthy and uniform sized seeds were packed in paper bags in petri dishes and placed into the irradiator and were exposed to X-rays of $75 \mathrm{KeV}$ at 5 and $10 \mathrm{sec}$ time periods.

For UV rays treatment, fifty healthy and uniform sized seeds of $P$. fistulous were presoaked in distilled water for two hours at room temperature and then seeds were immediately irradiated with UV C rays at time periods 1-2 $\mathrm{h}$ with wavelength of $280 \mathrm{~nm}$ using irradiation chamber equipped with UV C lamp (Milan Italy model; G15T8; $3.8 \mathrm{~J} / \mathrm{m}^{2}$ at $1 \mathrm{~m}$ of distance). After treatment, the seeds were planted in loamy soil and experiment was repeated twice thus total 150 seeds were used for each treatment.

Growth observations. The effect of chemical and physical mutagens on $\mathrm{M}_{1}$ plants of P. fistulous were observed, at an interval of one week during the growth of the plants. The growth parameters including, seeds germination (\%), seeds germination time, mutation frequency (\%), lethality (\%), stem length, leaf length, stem diameter, number of leaves, leaf surface area, flowering time, fruiting time and the number of fruits per plant, were observed during the study.

Germination (\%) of seeds. Germination test was carried out by sowing twenty five seeds on each seedbed and two seedbeds for each treatment. Seeds after absorbing moisture produced a normal root and then first leaf within five to ten days period was considered as germinated seeds. The germination value (\%) of each control and treated seeds was calculated according to Don (2005): 
Germination $(\%)=\frac{\text { No. of seeds germinated }}{\text { No. of seeds in soil }} \times 100$

Seed germination time. Total seed germination time was calculated by using the following formula:

$$
\text { Seed germination time }=\Sigma(\mathrm{d} \square \times \mathrm{n}) / \mathrm{S}
$$

where:

$\mathrm{d}=$ days from sowing; $\mathrm{n}=$ number of seedlings germinated on day "d"; $\mathrm{S}=$ the number of seedlings germinated (max: final day of total germinated seeds) (Ohba and Simak, 1961).

Lethality percentage (\%). Lethality \% of plants was calculated by using the following formula:

Lethality $(\%)=\frac{\text { No. of plants died }}{\text { Total no. of seeds germinated }} \times 100$

Calculation of mutation frequency (\%). Mutation frequency of the ethidium bromide, colchicine, UV rays and X-rays was calculated by the following formula:

$$
\text { Mutation frequency }(\%)=\mathrm{V} / \mathrm{P} \times 100
$$

where:

$\mathrm{M}=$ mutation frequency; $\mathrm{V}=$ viable mutants observed; $\mathrm{P}=$ total plants studied (Tah and Roychowdhury, 2011).

Measurement of stem length and diameter. The stem length was measured by using measuring scale or inch tape while the stem diameter was measured by using thread or scale.

\section{Measurement of leaf surface area and number of}

leaves. The leaves numbers were counted and leaf surface area was measured. Firstly, the petiole was removed from each control and treated as plant leaves. Leaf surface area was calculated by tracing the leaves of $P$. fistulosus onto $1 \mathrm{~cm}$, and $0.5 \mathrm{~cm}$ grid paper. For the calculation of surface area the whole blocks or squares located in the outline of leaves were counted and multiplied by the appropriate area of each grid size or square size (like for $1 \mathrm{~cm}$ grid paper it was multiplied by $1 \mathrm{~cm}^{2}$ and for $0.5 \mathrm{~cm}$ grid paper it was multiplied by $0.25 \mathrm{~cm}^{2}$ ). Then those squares which included just a small part of leaves outline were counted and divided by 2 since only part of the surface was included within the square. Now the whole square values and the partial square values were added up. As the grid size gets smaller, a better estimate of the true leaf surface area is determined. Using different graph sizes is a great lead to get accuracy and precision in surface area measurement (Gerber, 2014).

Harvesting of the fruit. Harvesting of fruit was started at different time periods in $8^{\text {th }}$ week after germination from each treatment. During harvesting mature fruits were cut from the fruit stalk using a knife.

Statistical analysis. The study plan was in randomized complete block design (RCBD) manner. Analysis was done on all the collected data. The mean of triplicates \pm standard deviation was calculated by using Microsoft Excel 2010 and used to assess differences between treatments, while the $p$-value $(0.05)$ was calculated by using IBM SPSS statistics 22.

\section{Results and Discussion}

Germination, growth and development of treated P. fistulosus plants are presented in Fig. 1-2. Analysis was applied to all measured data which showed significant $(\mathrm{p}<0.05)$ differences in terms of statistical analysis. The seed germination percentage was determined to be highest in case of control plants which showed that mutagenic treatments decreased the germination percentages but the second highest germination percentage $(55.0 \%)$ was in case of ethidium bromide $0.10 \%$ treated plants. Seed germination ( $\%$ ) was found to be highest in case of control which was $80 \%$ as compared to other treated plants, seed germination time was highest in case of control which was 3.5 days while the plants treated with X-rays $10 \mathrm{sec}$ took least time which was 1.60 days, mutation frequency (\%) was highest in the plants treated with colchicine $0.02 \%$ and X-rays $10 \mathrm{sec}$ which were $80 \%$, lowest lethality (\%) was in case of colchicine $0.02 \%$ and X-rays $10 \mathrm{sec}$ which was $20 \%$ but the growth of X-rays $10 \mathrm{sec}$ treated plants was stunted in starting weeks (Table 1).

Mutagenic frequency was found to be highest in the plants treated with colchicine $0.02 \%$ as reported by Tah and Roychowdhury (2011) that colchicine mutagenic action on Dianthus showed highest mutation frequency. The effect of ultraviolet B radiations on cucumber (Cucumis sativus) seeds resulted in decreased features (Yao et al., 2005) and almost similar results were observed in the present study with P. fistulosus. Increases in temperature have significant impact on the crop production due to global climate change, Sorghum bicolor (L). grown under higher temperatures resulted in more seed production and its germination (Prasad et al., 2006) as the tinda plants were grown in direct sun exposure. 
Ethidium bromide $0.10 \%$ treated plants showed the highest value of stem length $(77 \mathrm{~cm})$ while the lowest value of stem length was observed in case of X-rays $10 \mathrm{sec}$ treated plants which were $6.13 \mathrm{~cm}$ in the $11^{\text {th }}$ week (Table 2). The plants treated with colchicine $0.02 \%$ showed the larger leaf length which was $8 \mathrm{~cm}$ while the smaller length of the leaves were observed in X-rays $10 \mathrm{sec}$ treated plants which was $4.60 \mathrm{~cm}$ (Table 3). Ethidium bromide treated plants showed increase in leaves as the highest number of leaves $(36 \pm 2.64)$ per plant were observed in $0.1 \%$ ethidium bromide treated plants as compared to control plants (23.0 \pm 3.0 leaves per plant) while X-ray treatment significantly reduced the number of leaves $(1.66 \pm 0.57)$ per plant after $11^{\text {th }}$ week (Table 4). Thicker stem diameter was observed in control that is $3.1 \mathrm{~cm}$ while the least value of stem diameter was in case of X-rays $10 \mathrm{sec}$ treated plants which was $1.13 \mathrm{~cm}$ (Table 5).
Other researchers also observed drastic effects of $\mathrm{X}$-rays on plant growth, development and yield attributes. In chrysanthemum and carnation, form and colour in mutant flowers showed drastic effects when treated with ionizing radiations like $\mathrm{X}$-rays and gamma rays (Tanaka et al., 2010) that is the reason tinda seeds treated with X-rays $10 \mathrm{sec}$ retarded in their growth features during initial weeks. These results are also in agreement with the results of Ikram et al. (2015) that germination, shoot weight and root length was decreased when mungbean seeds were exposed to X-rays for 10 and $20 \mathrm{sec}$. Our results are also supported by Thapa (1999) that germination and seedling growth of $P$. wallichiana and $P$. kesiya were inhibited with increased exposure time of gamma rays. The largest leaf surface area was observed in case of colchicine $0.02 \%$ and similar results obtained in case of $0.1 \%$ colchicine treated Dianthus caryophyllus plants (Tah and

Table 1. Effect of chemical and physical mutagens on seed germination time, seed germination (\%), lethality (\%) and mutation frequency (\%) of $P$. fistulosus against control.

\begin{tabular}{|c|c|c|c|c|c|c|c|c|c|}
\hline \multirow[t]{2}{*}{ Parameters } & \multirow[t]{2}{*}{ Control } & \multicolumn{4}{|c|}{ Chemical mutagens } & \multicolumn{4}{|c|}{ Physical mutagens } \\
\hline & & $\begin{array}{l}\mathrm{EtBr} \\
(0.05 \%)\end{array}$ & $\begin{array}{l}\mathrm{EtBr} \\
(0.10 \%)\end{array}$ & $\begin{array}{l}\text { Col. } \\
(0.01 \%)\end{array}$ & $\begin{array}{l}\text { Col. } \\
(0.02 \%)\end{array}$ & $\begin{array}{l}\text { UV rays } \\
(1 \mathrm{~h})\end{array}$ & $\begin{array}{l}\text { UV rays } \\
(2 \mathrm{~h})\end{array}$ & $\begin{array}{l}\text { X-rays } \\
(5 \mathrm{sec})\end{array}$ & $\begin{array}{l}\text { X-rays } \\
(10 \mathrm{sec})\end{array}$ \\
\hline SGT (days) & 3.43 & 3.11 & 2.72 & 2.25 & 2.20 & 2.00 & 1.75 & 2.00 & 1.60 \\
\hline SG $(\%)$ & 80.0 & 45.0 & 55.0 & 40.0 & 25.0 & 15.0 & 20.0 & 15.0 & 25.0 \\
\hline $\mathrm{L}(\%)$ & 75.0 & 33.3 & 27.7 & 37.5 & 20.0 & 33.3 & 25.0 & 33.3 & 20.0 \\
\hline MF (\%) & ND & 66.6 & 54.5 & 62.5 & 80.0 & 66.6 & 75.0 & 66.6 & 80.0 \\
\hline
\end{tabular}

$\mathrm{SGT}=$ seed germination time; $\mathrm{SG}=$ seed germination $(\%) ; \mathrm{L}=$ lethality $(\%) ; \mathrm{MF}=$ mutation frequency $(\%)$; ND = not determined; $\mathrm{EtBr}=$ ethidium bromide; $\mathrm{Col}=$ colchicine; $\mathrm{UV}=$ ultraviolet rays.

Table 2. Effect of chemical and physical mutagens on stem length $(\mathrm{cm})$ of $P$. fistulosus against control (mean \pm S.D, $\mathrm{p}<0.05$ )

\begin{tabular}{|c|c|c|c|c|c|c|c|c|c|}
\hline \multirow{2}{*}{$\begin{array}{l}\text { Stem } \\
\text { length } \\
(\mathrm{cm})\end{array}$} & \multirow[t]{2}{*}{ Control } & \multicolumn{4}{|c|}{ Chemical mutagens } & \multicolumn{4}{|c|}{ Physical mutagens } \\
\hline & & $\begin{array}{l}\text { EtBr } \\
(0.05 \%)\end{array}$ & $\begin{array}{l}\text { EtBr } \\
(0.10 \%)\end{array}$ & $\begin{array}{l}\text { Col. } \\
(0.01 \%)\end{array}$ & $\begin{array}{l}\text { Col. } \\
(0.02 \%)\end{array}$ & $\begin{array}{l}\text { UV rays } \\
(1 \mathrm{~h})\end{array}$ & $\begin{array}{l}\text { UV rays } \\
(2 \mathrm{~h})\end{array}$ & $\begin{array}{l}\text { X-rays } \\
(5 \mathrm{sec})\end{array}$ & $\begin{array}{l}\text { X-rays } \\
(10 \mathrm{sec})\end{array}$ \\
\hline $1^{\text {st }}$ week & $3.83 \pm 0.28$ & $4.00 \pm 0.50$ & $3.66 \pm 0.25$ & $2.53 \pm 0.25$ & $1.90 \pm 0.26$ & $2.93 \pm 0.40$ & $2.20 \pm 0.34$ & $2.20 \pm 0.30$ & $3.06 \pm 0.11$ \\
\hline $2^{\text {nd }}$ week & $4.80 \pm 0.40$ & $4.20 \pm 0.45$ & $4.33 \pm 0.40$ & $3.10 \pm 0.26$ & $2.33 \pm 0.37$ & $3.73 \pm 0.25$ & $3.20 \pm 0.34$ & $2.76 \pm 0.50$ & $3.46 \pm 0.25$ \\
\hline $3^{\text {rd }}$ week & $6.36 \pm 0.45$ & $4.70 \pm 0.30$ & $4.70 \pm 0.36$ & $3.36 \pm 0.35$ & $2.73 \pm 0.35$ & $4.20 \pm 0.30$ & $3.83 \pm 0.35$ & $3.16 \pm 0.30$ & $4.36 \pm 0.41$ \\
\hline $4^{\text {th }}$ week & $8.03 \pm 0.15$ & $6.90 \pm 0.20$ & $7.50 \pm 0.30$ & $7.43 \pm 0.45$ & $5.80 \pm 0.20$ & $6.00 \pm 0.34$ & $4.96 \pm 0.60$ & $3.63 \pm 0.11$ & $4.73 \pm 0.15$ \\
\hline $5^{\text {th }}$ week & $14.2 \pm 0.30$ & $12.4 \pm 0.36$ & $9.46 \pm 0.40$ & $8.36 \pm 0.41$ & $6.86 \pm 0.15$ & $7.20 \pm 0.36$ & $5.86 \pm 0.35$ & $5.13 \pm 0.58$ & $4.75 \pm 0.40$ \\
\hline $6^{\text {th }}$ week & $21.3 \pm 0.45$ & $18.3 \pm 0.26$ & $19.9 \pm 0.41$ & $12.4 \pm 0.41$ & $11.0 \pm 0.90$ & $9.40 \pm 0.50$ & $9.03 \pm 0.15$ & $5.83 \pm 0.70$ & $4.76 \pm 0.30$ \\
\hline $7^{\text {th }}$ week & $25.3 \pm 0.45$ & $25.9 \pm 0.75$ & $23.8 \pm 0.49$ & $20.8 \pm 0.30$ & $19.9 \pm 0.65$ & $10.6 \pm 0.56$ & $9.90 \pm 0.30$ & $7.10 \pm 0.20$ & $5.46 \pm 0.56$ \\
\hline $8^{\text {th }}$ week & $30.9 \pm 0.65$ & $29.4 \pm 0.75$ & $31.7 \pm 0.55$ & $28.3 \pm 0.76$ & $20.8 \pm 0.90$ & $12.9 \pm 0.87$ & $11.0 \pm 0.35$ & $7.76 \pm 0.47$ & $5.73 \pm 0.61$ \\
\hline $9^{\text {th }}$ week & $38.5 \pm 0.45$ & $34.5 \pm 0.85$ & $40.8 \pm 0.30$ & $31.5 \pm 0.55$ & $28.2 \pm 0.76$ & $20.2 \pm 0.65$ & $16.3 \pm 1.15$ & $9.80 \pm 0.45$ & $5.93 \pm 0.51$ \\
\hline $10^{\text {th }}$ week & $48.9 \pm 1.87$ & $53.3 \pm 1.15$ & $71.7 \pm 2.02$ & $48.1 \pm 0.60$ & $43.2 \pm 1.35$ & $29.3 \pm 1.63$ & $19.6 \pm 1.01$ & $11.5 \pm 0.96$ & $6.10 \pm 0.45$ \\
\hline $11^{\text {th }}$ week & $49.5 \pm 1.11$ & $57.0 \pm 0.81$ & $77.0 \pm 1.20$ & $51.9 \pm 2.00$ & $49.8 \pm 1.23$ & $44.1 \pm 2.02$ & $37.8 \pm 1.76$ & $18.4 \pm 1.36$ & $6.13 \pm 0.45$ \\
\hline
\end{tabular}




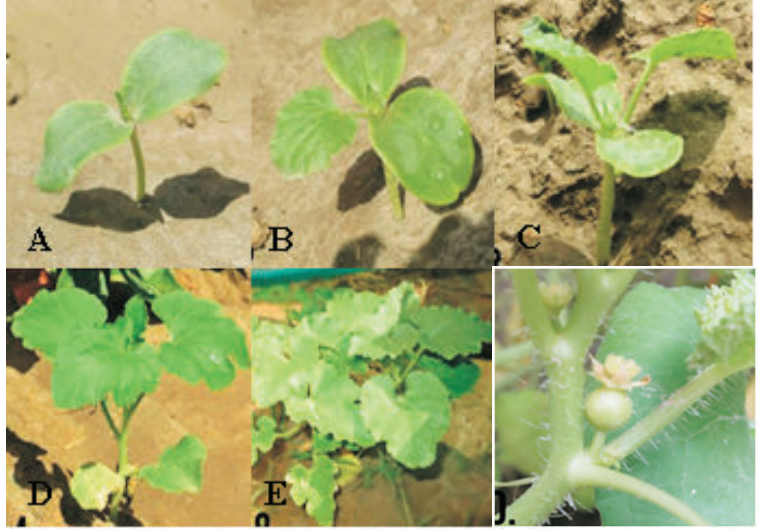

Fig. 1. Germination, growth and fruiting in $0.01 \%$ colchicine treated P. fistulosus.

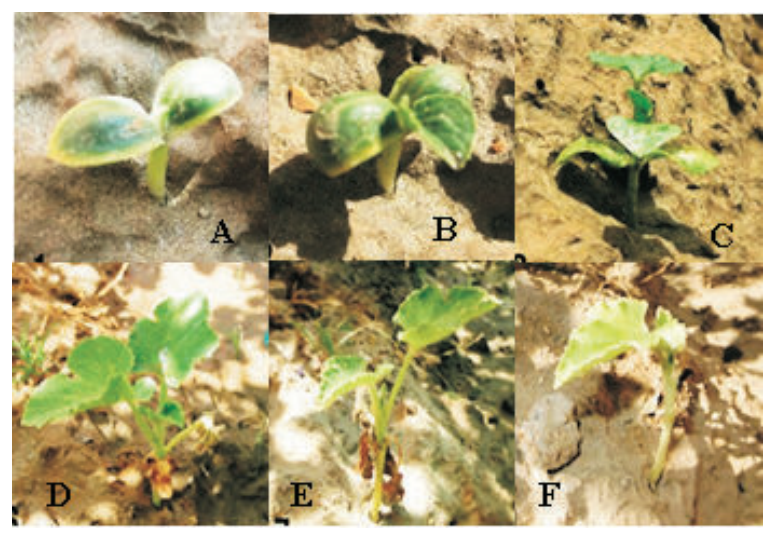

Fig. 2. Germination, growth and fruiting in X-rays (10 sec.) treated P. fistulosus.

Table 3. Effect of chemical and physical mutagens on leaf length $(\mathrm{cm})$ of $P$. fistulosus against control (mean \pm S.D, $\mathrm{p}<0.05)$

\begin{tabular}{|c|c|c|c|c|c|c|c|c|c|}
\hline \multirow{2}{*}{$\begin{array}{l}\text { Leaf } \\
\text { length } \\
\text { (cm) }\end{array}$} & \multirow[t]{2}{*}{ Control } & \multicolumn{4}{|c|}{ Chemical mutagens } & \multicolumn{4}{|c|}{ Physical mutagens } \\
\hline & & $\begin{array}{l}\text { EtBr } \\
(0.05 \%)\end{array}$ & $\begin{array}{l}\text { EtBr } \\
(0.10 \%)\end{array}$ & $\begin{array}{l}\text { Col. } \\
(0.01 \%)\end{array}$ & $\begin{array}{l}\text { Col. } \\
(0.02 \%)\end{array}$ & $\begin{array}{l}\text { UV rays } \\
(1 \mathrm{~h})\end{array}$ & $\begin{array}{l}\text { UV rays } \\
(2 \mathrm{~h})\end{array}$ & $\begin{array}{l}\text { X-rays } \\
(5 \mathrm{sec})\end{array}$ & $\begin{array}{l}\text { X-rays } \\
(10 \mathrm{sec})\end{array}$ \\
\hline $1^{\text {st }}$ week & & $2.23 \pm 0.20$ & $2.86 \pm 0.30$ & $1.43 \pm 0.25$ & $2.00 \pm 0.17$ & $2.90 \pm 0.20$ & $1.43 \pm 0.20$ & $2.73 \pm 0.15$ & $2.33 \pm 0.15$ \\
\hline $2^{\text {nd }}$ week & $3.46 \pm$ & $2.60 \pm 0.10$ & $2.93 \pm 0.35$ & $1.96 \pm 0.15$ & $2.13 \pm 0.20$ & $3.00 \pm 0.17$ & $3.30 \pm 0.34$ & $3.03 \pm 0.05$ & $2.43 \pm 0.05$ \\
\hline $3^{\text {rd }}$ week & $4.03 \pm 0.11$ & $3.23 \pm 0.25$ & $3.26 \pm 0.32$ & $4.13 \pm 0.20$ & $3.06 \pm 0.15$ & $3.10 \pm 0.26$ & $4.00 \pm 0.20$ & $3.53 \pm 0.05$ & $3.90 \pm 0.20$ \\
\hline $4^{\text {th }}$ week & $4.13 \pm 0.15$ & $4.40 \pm 0.17$ & $3.50 \pm 0.45$ & $4.36 \pm 0.28$ & $3.66 \pm 0.25$ & $3.43 \pm 0.11$ & $4.16 \pm 0.15$ & $3.60 \pm 0.10$ & $4.10 \pm 0.20$ \\
\hline $5^{\text {th }}$ week & $4.16 \pm 0.15$ & $4.56 \pm 0.25$ & $3.70 \pm 0.36$ & $4.56 \pm 0.30$ & $3.93 \pm 0.11$ & $3.56 \pm 0.11$ & $4.36 \pm 0.11$ & $3.73 \pm 0.15$ & $4.23 \pm 0.20$ \\
\hline $6^{\text {th }}$ week & $4.86 \pm 0.15$ & $4.73 \pm 0.11$ & $3.86 \pm 0.30$ & $4.73 \pm 0.25$ & $4.36 \pm 0.15$ & $3.90 \pm 0.30$ & $4.66 \pm 0.11$ & $3.80 \pm 0.17$ & $4.26 \pm 0.15$ \\
\hline $7^{\text {th }}$ week & $5.40 \pm 0.20$ & $5.03 \pm 0.15$ & $4.23 \pm 0.30$ & $5.23 \pm 0.37$ & $4.40 \pm 0.17$ & $4.36 \pm 0.32$ & $4.86 \pm 0.25$ & $4.50 \pm 0.20$ & $4.33 \pm 0.20$ \\
\hline $8^{\text {th }}$ week & $5.60 \pm 0.26$ & $5.16 \pm 0.15$ & $4.43 \pm 0.40$ & $5.70 \pm 0.17$ & $5.03 \pm 0.15$ & $4.66 \pm 0.35$ & $4.96 \pm 0.12$ & $5.00 \pm 0.40$ & $4.40 \pm 0.20$ \\
\hline $9^{\text {th }}$ week & $6.06 \pm 0.15$ & $6.43 \pm 0.45$ & $5.86 \pm 0.23$ & $5.83 \pm 0.05$ & $6.43 \pm 0.25$ & $4.96 \pm 0.05$ & $5.20 \pm 0.30$ & $5.01 \pm 0.26$ & $4.46 \pm 0.25$ \\
\hline $10^{\text {th }}$ week & $6.26 \pm 0.15$ & $6.86 \pm 0.15$ & $6.56 \pm 0.30$ & $6.03 \pm 0.05$ & $6.93 \pm 0.15$ & $6.93 \pm 0.15$ & $5.36 \pm 0.40$ & $6.43 \pm 0.35$ & $4.46 \pm 0.15$ \\
\hline $11^{\text {th }}$ week & $6.60 \pm 0.36$ & $7.83 \pm 0.20$ & $6.66 \pm 0.28$ & $6.80 \pm 0.55$ & $8.03 \pm 0.77$ & $7.90 \pm 0.40$ & $7.66 \pm 0.68$ & $6.56 \pm 0.30$ & $4.60 \pm 0.20$ \\
\hline
\end{tabular}

Table 4. Effect of chemical and physical mutagens on number of leaves of P. fistulosus against control (mean $\pm \mathrm{S}$.D, $\mathrm{p}<0.05)$

\begin{tabular}{|c|c|c|c|c|c|c|c|c|c|}
\hline \multirow{2}{*}{$\begin{array}{l}\text { Number } \\
\text { of leaves }\end{array}$} & \multirow[t]{2}{*}{ Control } & \multicolumn{4}{|c|}{ Chemical mutagens } & \multicolumn{4}{|c|}{ Physical mutagens } \\
\hline & & $\begin{array}{l}\text { EtBr } \\
(0.05 \%)\end{array}$ & $\begin{array}{l}\text { EtBr } \\
(0.10 \%)\end{array}$ & $\begin{array}{l}\text { Col. } \\
(0.01 \%)\end{array}$ & $\begin{array}{l}\text { Col. } \\
(0.02 \%)\end{array}$ & $\begin{array}{l}\text { UV rays } \\
(1 \mathrm{~h})\end{array}$ & $\begin{array}{l}\text { UV rays } \\
(2 \mathrm{~h})\end{array}$ & $\begin{array}{l}\text { X-rays } \\
(5 \mathrm{sec})\end{array}$ & $\begin{array}{l}\text { X-rays } \\
(10 \mathrm{sec})\end{array}$ \\
\hline & & $3.00 \pm 0.00$ & $2.33 \pm 0.57$ & $3.00 \pm 0.00$ & $2.33 \pm 0.57$ & $2.66 \pm 0.57$ & $2.00 \pm 0.00$ & $3.33 \pm 0.57$ & $2.33 \pm 0.57$ \\
\hline & & $5.33 \pm 0.15$ & $4.00 \pm 0.00$ & $3.33 \pm 0.57$ & $4.00 \pm 1.00$ & 6.00 & $4.66 \pm 0.57$ & $4.33 \pm 0.57$ & $4.00 \pm 0.00$ \\
\hline & $=0.57$ & $6.33 \pm 0.57$ & $4.66 \pm 0.57$ & $4.00 \pm 1.00$ & $5.33 \pm 1$ & $6.66 \pm$ & $5.33 \pm 0.57$ & $4.33 \pm 0.57$ & $4.33 \pm 0.57$ \\
\hline & $7.66 \pm 0.57$ & $9.00 \pm 0.00$ & $7.33 \pm 0.57$ & $5.00 \pm 1.00$ & $6.33 \pm 1.15$ & $7.00 \pm 0.00$ & $7.33 \pm 0.57$ & $6.33 \pm 0.57$ & $4.33 \pm 0.57$ \\
\hline $6^{\text {th }}$ week & $10.6 \pm 0.57$ & $11.3 \pm 1.15$ & $8.66 \pm 1.15$ & $7.66 \pm 0.57$ & $7.00 \pm 1.00$ & $7.66 \pm 0.57$ & $8.00 \pm 0.00$ & $8.00 \pm 1.00$ & $4.33 \pm 0.57$ \\
\hline $7^{\text {th }}$ week & & $13.6 \pm 1.52$ & $10.6 \pm 0$ & $12.0 \pm 1$ & $8.33 \pm$ & $8.33 \pm 0.57$ & $8.33 \pm 0.57$ & $8.33 \pm 1.15$ & $4.66 \pm 0.57$ \\
\hline $3^{\text {th }}$ week & $14.6 \pm 1.52$ & $17.3 \pm 0.57$ & $14.6 \pm 0.57$ & $14.6 \pm 1.15$ & $17.3 \pm 1$ & $8.35 \pm 0.57$ & $8.33 \pm 0.57$ & $5.66 \pm 0.57$ & $2.33 \pm 0.57$ \\
\hline $9^{\text {th }}$ week & $25.0 \pm 2.00$ & $32.0 \pm 2.00$ & $36.0 \pm 1.73$ & $21.0 \pm 1.00$ & $30.0 \pm 1.00$ & $17.3 \pm 1.15$ & $23.3 \pm 0.57$ & $8.00 \pm 1.00$ & $2.33 \pm 0.57$ \\
\hline & $22.0 \pm 1.73$ & $28.0 \pm 1.00$ & $33.0 \pm 3.00$ & $19.3 \pm 0.57$ & $31.3 \pm 1.52$ & & $25.3 \pm 1.52$ & $8.33 \pm 0.57$ & $2.33 \pm 0.57$ \\
\hline $11^{\text {th }}$ week & $23.0 \pm 3.00$ & $28.3 \pm 1.52$ & $36.0 \pm 2.64$ & $22.0 \pm 1.00$ & $33.0 \pm 1.73$ & $29.6 \pm 2.08$ & $25.3 \pm 0.57$ & $8.66 \pm 0.57$ & $1.66 \pm 0.57$ \\
\hline
\end{tabular}


Roychowdhury, 2011). Our results are also in agreement with Essel et al. (2015) that colchicine stimulated the vegetative growth with increased plant height, number of branches and leaves in cowpea. The largest leaf surface area was in case of colchicine $0.02 \%$ which was $51.1 \mathrm{~cm}^{2}$ while the smallest surface area was in

Table 5. Effect of chemical and physical mutagens on stem diameter (cm) of $P$. fistulosus against control (mean \pm S.D, $\mathrm{p}<0.05)$

\begin{tabular}{|c|c|c|c|c|c|c|c|c|c|}
\hline \multirow{2}{*}{$\begin{array}{l}\text { Stem } \\
\text { diameter } \\
(\mathrm{cm})\end{array}$} & \multirow[t]{2}{*}{ Control } & \multicolumn{4}{|c|}{ Chemical mutagens } & \multicolumn{4}{|c|}{ Physical mutagens } \\
\hline & & $\begin{array}{l}\text { EtBr } \\
(0.05 \%)\end{array}$ & $\begin{array}{l}\mathrm{EtBr} \\
(0.10 \%)\end{array}$ & $\begin{array}{l}\text { Col. } \\
(0.01 \%)\end{array}$ & $\begin{array}{l}\text { Col. } \\
(0.02 \%)\end{array}$ & $\begin{array}{l}\text { UV rays } \\
(1 \mathrm{~h})\end{array}$ & $\begin{array}{l}\text { UV rays } \\
(2 \mathrm{~h})\end{array}$ & $\begin{array}{l}\text { X-rays } \\
(5 \mathrm{sec})\end{array}$ & $\begin{array}{l}\text { X-rays } \\
(10 \mathrm{sec})\end{array}$ \\
\hline $1^{\text {st }}$ week & $0.66 \pm 0.05$ & $0.60 \pm 0.00$ & $0.50 \pm 0.00$ & $0.43 \pm 0.11$ & $0.73 \pm 0.05$ & $0.66 \pm 0.05$ & $0.43 \pm 0.11$ & $0.73 \pm 0.11$ & $0.66 \pm 0.15$ \\
\hline $2^{\text {nd }}$ week & $0.66 \pm 0.15$ & $0.63 \pm 0.05$ & $0.76 \pm 0.15$ & $0.76 \pm 0.15$ & $0.76 \pm 0.05$ & $0.80 \pm 0.17$ & $0.63 \pm 0.11$ & $0.86 \pm 0.15$ & $0.73 \pm 0.05$ \\
\hline $3^{\text {rd }}$ week & $0.80 \pm 0.10$ & $0.76 \pm 0.05$ & $0.79 \pm 0.10$ & $0.76 \pm 0.11$ & $0.76 \pm 0.15$ & $0.83 \pm 0.15$ & $0.63 \pm 0.05$ & $0.90 \pm 0.10$ & $0.80 \pm 0.10$ \\
\hline $4^{\text {th }}$ week & $0.93 \pm 0.05$ & $0.93 \pm 0.05$ & $1.06 \pm 0.11$ & $0.93 \pm 0.05$ & $0.93 \pm 0.15$ & $0.86 \pm 0.15$ & $0.83 \pm 0.20$ & $0.90 \pm 0.10$ & $0.83 \pm 0.11$ \\
\hline $5^{\text {th }}$ week & $1.43 \pm 0.25$ & $1.06 \pm 0.11$ & $1.16 \pm 0.15$ & $1.03 \pm 0.15$ & $0.96 \pm 0.15$ & $1.20 \pm 0.20$ & $0.90 \pm 0.10$ & $0.93 \pm 0.11$ & $0.86 \pm 0.15$ \\
\hline $6^{\text {th }}$ week & $1.83 \pm 0.11$ & $1.26 \pm 0.30$ & $1.56 \pm 0.35$ & $1.60 \pm 0.34$ & $1.26 \pm 0.25$ & $1.66 \pm 0.25$ & $0.96 \pm 0.05$ & $1.46 \pm 0.15$ & $0.96 \pm 0.05$ \\
\hline $7^{\text {th }}$ week & $1.96 \pm 0.05$ & $2.00 \pm 0.30$ & $1.70 \pm 0.43$ & $1.80 \pm 0.20$ & $1.26 \pm 0.25$ & $1.66 \pm 0.25$ & $1.23 \pm 0.40$ & $1.63 \pm 0.35$ & $1.03 \pm 0.15$ \\
\hline $8^{\text {th }}$ week & $2.06 \pm 0.20$ & $2.06 \pm 0.32$ & $1.83 \pm 0.32$ & $1.93 \pm 0.11$ & $1.26 \pm 0.25$ & $1.70 \pm 0.30$ & $1.93 \pm 0.45$ & $1.80 \pm 0.43$ & $1.13 \pm 0.11$ \\
\hline $9^{\text {th }}$ week & $2.40 \pm 0.20$ & $2.00 \pm 0.17$ & $1.83 \pm 0.11$ & $2.06 \pm 0.11$ & $1.90 \pm 0.26$ & $1.70 \pm 0.17$ & $2.16 \pm 0.37$ & $2.00 \pm 0.26$ & $1.13 \pm 0.11$ \\
\hline $10^{\text {th }}$ week & $2.60 \pm 0.25$ & $2.16 \pm 0.15$ & $2.23 \pm 0.20$ & $2.13 \pm 0.15$ & $2.36 \pm 0.30$ & $2.10 \pm 0.36$ & $2.40 \pm 0.36$ & $2.05 \pm 0.20$ & $1.13 \pm 0.20$ \\
\hline $11^{\text {th }}$ week & $3.16 \pm 0.20$ & $2.63 \pm 0.20$ & $2.83 \pm 0.15$ & $2.56 \pm 0.11$ & $2.93 \pm 0.25$ & $2.20 \pm 0.30$ & $2.70 \pm 0.20$ & $2.53 \pm 0.40$ & $1.13 \pm 0.20$ \\
\hline
\end{tabular}

Table 6. Effect of chemical and physical mutagens on leaf surface area $\left(\mathrm{cm}^{2}\right)$ of $P$. fistulosus against control (mean \pm S.D, $\mathrm{p}<0.05$ )

\begin{tabular}{|c|c|c|c|c|c|c|c|c|c|}
\hline \multirow{2}{*}{$\begin{array}{l}\text { Leaf } \\
\text { surface } \\
\text { area }\left(\mathrm{cm}^{2}\right)\end{array}$} & \multirow[t]{2}{*}{ Control } & \multicolumn{4}{|c|}{ Chemical mutagens } & \multicolumn{4}{|c|}{ Physical mutagens } \\
\hline & & $\begin{array}{l}\text { EtBr } \\
(0.05 \%)\end{array}$ & $\begin{array}{l}\text { EtBr } \\
(0.10 \%)\end{array}$ & $\begin{array}{l}\text { Col. } \\
(0.01 \%)\end{array}$ & $\begin{array}{l}\text { Col. } \\
(0.02 \%)\end{array}$ & $\begin{array}{l}\text { UV rays } \\
(1 \mathrm{~h})\end{array}$ & $\begin{array}{l}\text { UV rays } \\
(2 \mathrm{~h})\end{array}$ & $\begin{array}{l}\text { X-rays } \\
(5 \mathrm{sec})\end{array}$ & $\begin{array}{l}\text { X-rays } \\
(10 \mathrm{sec})\end{array}$ \\
\hline $1^{\text {st }}$ week & $10.4 \pm 0.83$ & $12.9 \pm 0.62$ & $10.2 \pm 0.15$ & $15.0 \pm 0.20$ & $14.9 \pm 0.05$ & $15.1 \pm 0.41$ & $11.9 \pm 0.75$ & $8.76 \pm 0.40$ & $7.13 \pm 0.32$ \\
\hline $2^{\text {nd }}$ week & $15.5 \pm 1.45$ & $17.3 \pm 1.19$ & $12.7 \pm 0.75$ & $12.7 \pm 0.46$ & $22.3 \pm 0.41$ & $15.2 \pm 1.01$ & $14.5 \pm 1.13$ & $12.2 \pm 0.68$ & $8.30 \pm 0.20$ \\
\hline $3^{\text {rd }}$ week & $17.3 \pm 0.50$ & $19.2 \pm 0.43$ & $14.0 \pm 0.20$ & $24.0 \pm 0.63$ & $24.8 \pm 0.26$ & $15.7 \pm 0.81$ & $17.7 \pm 1.11$ & $14.9 \pm 0.65$ & $8.97 \pm 0.25$ \\
\hline $4^{\text {th }}$ week & $27.6 \pm 0.65$ & $29.9 \pm 0.36$ & $23.8 \pm 0.35$ & $27.1 \pm 0.32$ & $28.8 \pm 0.32$ & $22.0 \pm 0.11$ & $26.7 \pm 0.68$ & $20.0 \pm 0.20$ & $10.2 \pm 0.64$ \\
\hline $5^{\text {th }}$ week & $28.9 \pm 0.05$ & $30.3 \pm 0.61$ & $25.1 \pm 0.32$ & $29.5 \pm 0.89$ & $31.2 \pm 1.12$ & $27.4 \pm 0.68$ & $29.5 \pm 0.51$ & $20.1 \pm 0.15$ & $10.5 \pm 0.63$ \\
\hline $6^{\text {th }}$ week & $29.7 \pm 0.50$ & $31.1 \pm 0.49$ & $27.3 \pm 0.58$ & $28.7 \pm 0.64$ & $39.0 \pm 0.20$ & $41.3 \pm 0.15$ & $38.7 \pm 0.87$ & $20.1 \pm 0.41$ & $11.0 \pm 0.51$ \\
\hline $7^{\text {th }}$ week & $32.2 \pm 0.43$ & $34.9 \pm 0.17$ & $28.9 \pm 0.45$ & $30.9 \pm 0.15$ & $39.5 \pm 1.30$ & $44.6 \pm 1.09$ & $39.6 \pm 0.65$ & $21.0 \pm 0.05$ & $11.7 \pm 0.34$ \\
\hline $8^{\text {th }}$ week & $33.1 \pm 0.52$ & $36.0 \pm 0.05$ & $29.8 \pm 0.15$ & $31.6 \pm 0.76$ & $41.1 \pm 1.00$ & $46.8 \pm 0.76$ & $40.8 \pm 0.75$ & $21.2 \pm 0.25$ & $11.9 \pm 0.40$ \\
\hline $9^{\text {th }}$ week & $35.6 \pm 0.55$ & $37.2 \pm 0.43$ & $30.6 \pm 0.35$ & $34.4 \pm 0.45$ & $42.0 \pm 0.20$ & $47.6 \pm 1.09$ & $43.0 \pm 1.69$ & $22.0 \pm 0.20$ & $12.2 \pm 0.26$ \\
\hline $10^{\text {th }}$ week & $38.4 \pm 0.50$ & $39.1 \pm 0.32$ & $33.8 \pm 0.98$ & $37.7 \pm 1.16$ & $48.9 \pm 0.85$ & $48.9 \pm 1.24$ & $46.0 \pm 1.80$ & $22.2 \pm 0.26$ & $12.7 \pm 0.20$ \\
\hline $11^{\text {th }}$ week & $39.5 \pm 0.51$ & $50.8 \pm 0.90$ & $34.2 \pm 0.92$ & $43.2 \pm 2.34$ & $51.1 \pm 0.69$ & $49.6 \pm 1.47$ & $44.3 \pm 1.53$ & $22.4 \pm 0.37$ & $12.9 \pm 0.05$ \\
\hline
\end{tabular}

Table 7. Effect of chemical and physical mutagens on flowering time, fruiting time and number of fruits of $P$. fistulosus against control (mean \pm S.D)

\begin{tabular}{|c|c|c|c|c|c|c|c|c|c|}
\hline \multirow[t]{2}{*}{ Parameters } & \multirow[t]{2}{*}{ Control } & \multicolumn{4}{|c|}{ Chemical mutagens } & \multicolumn{4}{|c|}{ Physical mutagens } \\
\hline & & $\begin{array}{l}\mathrm{EtBr} \\
(0.05 \%)\end{array}$ & $\begin{array}{l}\text { EtBr } \\
(0.10 \%)\end{array}$ & $\begin{array}{l}\text { Col. } \\
(0.01 \%)\end{array}$ & $\begin{array}{l}\text { Col. } \\
(0.02 \%)\end{array}$ & $\begin{array}{l}\text { UV rays } \\
(1 \mathrm{~h})\end{array}$ & $\begin{array}{l}\text { UV rays } \\
(2 \mathrm{~h})\end{array}$ & $\begin{array}{l}\text { X-rays } \\
(5 \mathrm{sec})\end{array}$ & $\begin{array}{l}\text { X-rays } \\
(10 \mathrm{sec})\end{array}$ \\
\hline FLT (days) & $44.5 \pm 0.70$ & $31.0 \pm 1.41$ & $42.5 \pm 0.70$ & $38.5 \pm 2.12$ & $49.0 \pm 1.41$ & --- & --- & --- & --- \\
\hline FRT (days) & --- & --- & $60.0 \pm 1.41$ & $42.5 \pm 2.12$ & $69.0 \pm 2.82$ & --- & --- & --- & --- \\
\hline NF & --- & --- & $2.50 \pm 0.70$ & $4.00 \pm 1.41$ & $2.50 \pm 0.70$ & --- & --- & --- & --- \\
\hline
\end{tabular}

FLT $=$ flowering time, $\mathrm{FRT}=$ fruiting time, $\mathrm{NF}=$ number of fruits. 
X-rays $10 \mathrm{sec}$ which was $12.9 \mathrm{~cm}^{2}$ (Table 6). Mutagenic effects on plants features may produce resistance in many susceptible crops and their yield improvement (Al-Qurainy and Khan, 2009). Mensah et al. (2007) also obtained higher yield or number of fruits in low concentration of colchicine $(0.01 \%)$ in Sesame indicum L. The minimum flowering time was observed in ethidium bromide $0.05 \%$ treated plants which was 31 days, while the flowering time increased in case of colchicine $0.02 \%$ treated plants which was 49 days but the plants with treatments such as UV (1 h and $2 \mathrm{~h})$ and $\mathrm{X}$-rays $(5 \mathrm{sec}$ and $10 \mathrm{sec}$ ) did not show any flowering, while fruiting time was minimum in case of colchicine $0.01 \%$ that was 42.5 days and maximum in colchicine $0.02 \%$ treated plants but the plants treated with ethidium bromide $0.05 \%$, UV ( $1 \mathrm{~h}$ and $2 \mathrm{~h}$ ), X-rays ( $5 \mathrm{sec}$ and $10 \mathrm{sec}$ ) and control did not show any fruiting. The highest number of fruits was observed in colchicine $0.01 \%$ which was 4 in number, while lowest number of fruits was observed in case of ethidium bromide $0.01 \%$ and colchicine $0.02 \%$ treated plants. On the other hand, control plants were flowered but failed to fruit due to high temperature (Table 7). These results revealed that treatment of $P$. fistulosus seeds with colchicine $(0.01$ and $0.02 \%)$ and ethidium bromide $(0.1 \%)$ may be beneficial that increased heat tolerance and thus made flowering and fruiting even when temperature increased to $45^{\circ} \mathrm{C}$ during the month of June.

These results are in agreement with Dhakhanamoorthy et al. (2010) that early flowering and fruit maturity may be due to the physiological changes caused by mutagen. The secondary compounds such as phenolic acids, antioxidants, reducing power, ascorbic acids, etc. were significantly enhanced in colchicine treated $P$. fistulosus plants as compared to other mutagens treated plants (data not shown). The fruits of P. fistulosus are used as vegetable while the leaves are the agriculture wastes. The secondary metabolites of the plants have commercial interest both in research and pharmaceuticals for the manufacturing of the effective drugs. The growth features can be improved or modified under the influence of mutagenic treatment. The present study has provided evidence on the induction of mutations causing genetic variability associated with growth, reproductive and yield parameters of tinda (Praecitrullus fistulosus). Thus, genetic variability caused by mutation induction can be effectively utilized for modifying mutant strains possessing desirable and novel attributes.

\section{Conclusion}

The results of mutagen treatment of $P$. fistulosus revealed ethidium bromide and colchicine treatment of seeds improved germination, growth and yield attributes in high temperature while UV and X-rays treatment showed opposing results of these attributes. Similarly $0.01 \%$ colchicine may be used for the improvement of growth, reproductive and yield parameters in P. fistulosus.

\section{References}

Adamu, A.K., Aliyu, H. 2007. Morphological effects of sodium azide on tomato (Lycopersicon esculentum) Mill. Science World Journal, 2: 9-12.

Akwaowo, E.U., Ndon, B.A., Etuk, E.U. 2000. Minerals and antinutrients in fluted pumpkin (Telfairia occidentalis Hook f.). Food Chemical, 70: 235-240.

Al-Qurainy, F., Khan, S. 2009. Mutagenic effects of sodium azide and its application in crop improvement. World Applied Science Journal, 6: 1589-1601.

Amano, E. 2006. Use of induced mutants in rice breeding in Japan. Plant Mutation Reports, 1: 21-24.

Asif, M., Saleem, M.F., Anjum, S.A., Wahid, M.A., Bilal, M.F. 2013. Effect of nitrogen and zinc sulphate on growth and yield of maize (Zea Mays L.). Journal of Agricultural Research, 51: 455-464.

Bourgaud, F., Gravot, A., Milesi, S., Gontier, E. 2001. Production of plant secondary metabolites: a historical perspective. Plant Science, 165: 839-851.

Chopra, V.L. 2005. Mutagenesis: Investigating the process and processing the outcome for crop improvement. Current Science, 89: 353-359.

Dhakhanamoorthy, D., Selvaraj, R., Chidambaram, A. 2010. Physical and chemical mutagenesis in Jatropha curcas L. to induce variability in seed germination, growth and yield traits. Romanian Journal of Plant Biology, 55: 113-125.

Dixit, Y., Kar, A. 2010. Protective role of three vegetable peels in alloxan induced diabetes mellitus in male mice. Plant Foods for Human Nutrition, 65: 284289.

Don, R. 2005. Germination rounding procedure, ISTA germination committee. International Seed Testing Association, 130: 46-47.

Essel, E., Asante, I.K., Laing, E. 2015. Effect of colchicine treatment on seed germination, plant growth and yield traits of cowpea (Vigna unguiculata (L.) Walp. Canadian Journal of Pure and Applied Sciences, 9: 3573-3576.

Gautam, S., Singh, P., Shivha, Y. 2011. Praecitrullus 
fistulosus: A miraculous plant. Asian Journal of Pharmaceutical Technology, 1: 9-12.

Gerber, F., Marion, R., Olioso, A., Jacquemoud, S., daLuz, B.R., Fabre, S. 2011. Modeling directionalhemispherical reflectance and transmittance of fresh and dry leaves from $0.4 \mu \mathrm{m}$ to $5.7 \mu \mathrm{m}$ with the PROSPECT-VISIR model. Remote Sensing of Environment, 115: 404-414.

Ikram, N., Dawar, S., Imtiaz, F. 2015. X-rays treated leguminous seeds in combination with wild plant powder for the promotion of growth and control of root rot fungi. Journal of Plant Pathology and Microbiology, S3: 003. doi:10.4172/2157-7471.S3003

Islam, M.R., Prodhan, A.K., Islam, M.O., Uddin, M.K. 2010. Effect of plant growth regulator (GABA) on morphological characters and yield of black gram (Vigna mungo L.). Journal of Agricultural Research, 48: 73-80.

Jimoh, F.O., Oladiji, A.T. 2005. Preliminary studies on Piliostigma thonningii seeds: Proximate analysis, mineral composition and phytochemical screening. African Journal of Biotechnology, 4: 1439-1442.

Katerova, Z., Ivanov, S., Prinsen, E., Van Onckelen, H., Alexieva, V., Azmi, A. 2009. Low doses of ultraviolet-B or ultraviolet-C radiation affect $\mathrm{ACC}$, ABA and IAA levels in young pea plants. Biological Plantarium, 53: 365-368.

Kharkwal, M.C.. Shu, Q.Y. 2009. The role of induced mutations in World Food Security. In: Induced Plant Mutations in the Genomics Era, pp. 33-38, Shu Q. Y. (ed.), Food and Agriculture Organization of the United Nations, Rome, Italy.

Koornneeff, M., Dellaert, L.W.M., Van der Veen, J.H. 1982. EMS-and radiation-induced mutation frequencies at individual loci in Arabidopsis thaliana (L.) Heynh. Mutation Research, 93: 109-123.

Levi, A., Harris, K.R., Wechter, W.P., Kousik, C.S., Thies, J.A. 2010. DNA markers and pollen morphology reveal that Praecitrullus fistulosus is more closely related to Benincasa hispida than to Citrullus spp. Genetic Resources and Crop Evolution, 57: 1191-1205.

Mensah, J.K., Obadoni, B.O., Akomeah, P.A., Ikhajiagbe, B., Ajibolu, J. 2007. The effects of sodium azide and colchicine treatments on morphological and yield traits of sesame seed (Sesame indicum L.). African Journal of Biotechnology, 6: 534-538.

Ohba, K., Simak, M. 1961. Effect of X-rays on seeds of Scots pine from different provenances (Pinus silvestris L.). Silvae Genetica, 10: 84-90.

Palchaudhuri, R., Hergenrother, P.J. 2007. DNA as a target for anticancer compounds: methods to determine the mode of binding and the mechanism of action. Current Opinion Biotechnology, 18: 497-503.

Prakash, D., Pal, M. 1991. Nutritional and antinutritional comparison of vegetable and grain Amaranthus leaves. Journal of Science and Food and Agricultural, 57: 573-585.

Prasad, P.V., Boote, K.J., Allen, L.H. 2006. Adverse high temperature effects on pollen viability, seedset, seed yield and harvest index of grain-sorghum Sorghum bicolor (L.) Moench are more severe at elevated carbon dioxide due to higher tissue temperatures. Agriculture and Forest Meteorology, 139: 237-251.

Ravelli, R.B., Gigant, B., Curmi, P.A., Jourdain, I., Lachkar, S., Sobel, A., Knossow, M. 2004. Insight into tubulin regulation from a complex with colchicine and a stathmin-like domain. Nature, 428: 198-202.

Sameh, A.S.A., Kumar, A.P., Rao, B.S., Singh, R.P. 2006. Biodegradation of $\gamma$-sterilised biomedical polyolefins under composting and fungal culture environments. Polymer Degradation and Stability, 91: 1105-1116.

Siddiqui, A., Dawar, S., Zaki, J.M., Hamid, N. 2011. Role of ultra violet (UV-C) radiation in the control of root infecting fungi on groundnut and mung bean. Pakistan Journal of Botany, 43: 2221-2224.

Song, H.P., Kim, D.H., Jo, C., Lee, C.H., Kim, K.S. 2006. Effect of gamma irradiation on the microbiological quality and antioxidant activity of fresh vegetable juice. Food Microbiology, 23: 372-378.

Sujatha, V.S., Seshadri, V.S. 1989. Taxonomic position of round melon (Praecitrullus fistulosus). Cucurbit Genetics Cooperative Reports, 12: 86-88.

Sutherland, B.M., Bennett, P.V., Sidorkina, O., Laval, J. 2000. Clustered DNA damages induced in isolated DNA and in human cell by low doses of ionizing radiation. Proceedings of the Natural Academy of Sciences of the United States of America, 97: 103108.

Tah, J., Roychowdhury, R. 2011. Chemical mutagenic action on seed germination and related agro-metrical traits in M1 Dianthus generation. Current Botany, 2: 19-23.

Tanaka, A., Shikazono, N., Hase, Y. 2010. Studies on biological effects of ion beams on lethality, molecular 
nature of mutation, mutation rate, and spectrum of mutation phenotype for mutation breeding in higher plants. Journal of Radiation Research, 51: 223-233.

Taylor, J.S. 2006. Structure and properties of DNA photoproducts. In: DNA Damage Recognition, W. Siede, Y. W. Kow, P. W. Doetsch (eds). pp. 6794, Taylor and Francis Group, New York, USA.

Thapa, C.B. 1999. Effect of acute exposure of gamma rays on seed germination Pinus kesiya Gord and P. wallichiana A.B. Jacks. Botanica Orientalis Journal of Plant Science, 1: 120-121.

Van Harten, A.M. 1998. Mutation Breeding: Theory and Practical Applications, Cambridge University Press, Cambridge, UK.

Yang, W., Xu, X., Duan, L., Luo, Q., Chen, S., Zeng, S., Liu, Q. 2011. High-throughput measurement of rice tillers using a conveyor equipped with X-ray computed tomography. Review Scientific Instruments, 82: 025102.

Yao, Y., Li, Y., Yang, Y., Li C. 2005. Effect of seed pretreatment by magnetic field on the sensitivity of cucumber (Cucumis sativus) seedlings to ultraviolet-B radiation. Environmental and Experimental Botany, 54: 286-294. 